\title{
Response Time Minimization Task Scheduling Algorithm
}

\author{
M. Hemamalini \\ Research Scholar, Bharathidasan University \\ Assistant Professor, \\ Department of Computer Science, \\ A.V.C College (Autonomous), Mannampandal, \\ Mayiladuthurai, India
}

\author{
M. V. Srinath, PhD \\ Director, Department of MCA, \\ Sengamala Thayaar Educational \\ Trust Women's College, \\ Mannargudi, \\ India
}

\begin{abstract}
Computational grid has been measured as the best model for managing large scale distributed system having geographically owed resources. Load balancing algorithms are important in the research of network applications. The proposed algorithm minimizes the average execution time and response time. The proposed Response Time Minimization algorithm is implemented with Cloudanalyst toolkit which can simulate a decentralized module. The Cloudanalyst toolkit abstracts the features and behavior of complex fundamental elements such as tasks, resources and users. The proposed algorithm presents service namely resource discovery. The proposed Response Time Minimization Approach is compared with Round Robin Algorithm and equally spread execution algorithm. The main objective of the Response time minimization algorithm is to share the load to the available Virtual Machine (VM) efficiently in order to improve the response time, data processing time and minimize delay time. The performance analysis indicates that the response time of the proposed task scheduling algorithm is much better than the Round Robin Algorithm and equally spread execution algorithm. Results support the proposed approach.
\end{abstract}

\section{Keywords}

Task scheduling, Response Time, virtual Machine Data center and Load balancing.

\section{INTRODUCTION}

In order to meet the business requirements, in terms of Quality of Service (QoS), cloud service provisioning is coordinated by Service Level Agreements (SLAs) resulting in contracts between clients and providers that articulate the price and the Quality of service levels required during the service provisioning, and the penalties for SLA violations. In this case, performance evaluation plays a key role and allows the system managers to review the impact of resource management strategies on the data center functioning and to calculate the corresponding costs/benefits. The greater number of parameter that have to be investigated. So simulation does not allow the comprehensive analyses of the system performance [1]. To overcome the issues, the proposed Response Time Minimization Scheduling Model which demonstrates the above mentioned features and capturing the key concepts of an IAAS cloud system. The proposed model is adequate and scalable to represent systems composed of thousands of resources and it makes possible to represent both physical and virtual resources make use of the cloud specific concepts such as the infrastructure elasticity. This also reduces the bandwidth issues and maintains job arrival process and System Queue. This model easily evaluates the data centre performance and response time. This model is applicable for both large and small infrastructure, which helps to monitor the user and data centre regionally and globally.

\subsection{Features}

- Efficient Scheduling Model is efficient to resolve the earlier mentioned issues. It reduces the request response time and error during data processing.

- The proposed Scheduling Model composes thousands of resources and is flexible to represent different policies and cloud-specific strategies

- The proposed design is to support, monitor and evaluate data from various regions.

- The proposed model allows system analyzes the better solution with respect to an established goal and to favourably set the system parameters.

- The proposed design has less energy consumption and cost effective.

\section{RELATED WORK}

Pushtikant Malviya et al. [2] have proposed a very efficient method of resource provisioning to minimize the power consumption in virtual cloud environment. The researcher suggested many techniques such as Dynamic Voltage Frequency Scaling Technique (DVFS) to save energy. Authors tried the allocation of various resources or Migration of Virtual Machine Techniques in diverse practical conditions to make the cloud environmental friendly. The main attention of this research was to do effective and efficient resource provisioning based on migration of VM's.

Akhil [3] suggested various soft computing techniques for load balancing. These methods are very proficient for solving NP-complete problems and load balancing is achieved. This paper analyzes the performance of different soft computing techniques by using fixed parameters and states the advantages and drawbacks among them.

Load balancing algorithm is used to share the load among all the available resources [4]. The algorithm attempts to improve the utilization of resources with light load and freeing the resources with heavy load. The two factors such as Execution Time and Memory requirement are used for load balancing and effective Utilization of resources [5].

T. Kokila vani [6] proposed Load Balanced Min-Min scheduling algorithm which produces better results than minmin scheduling algorithm. It reduces the makespan and balance the load.

M.Hemamalini [7] proposed the method memory constrained load shared minimum execution Time Grid Meta task scheduling algorithm is used to balance the load and reduces the makespan based on memory requirement of the task. Resource Utilization can be calculated and analyzes the performance with MET Meta scheduling algorithm. 
Anton Beloglazov et al. [8] proposed the method to manage the data centers efficiently in a cloud environment. This paper describes the heuristic algorithm for the energy aware resource allocation and architectural principles for energyefficient management in a cloud environment. In their research work they also proposed the heuristic techniques and meta task scheduling algorithms for energy effective resource distribution based on the QoS and power usage characteristics.

Bharti Wadhwa [9] analyzes the performance of various approaches to save energy consumption in green cloud computing. This paper describes the work of different researchers to reduce the carbon footprints emissions by the method used for virtualization, VM migration and scheduling.

Jens Buysse et al. [10] described a two-step approach to minimize the energy consumption in optical clouds. This routing and scheduling algorithm selects unused nodes and turnoff these nodes. In the first step it focuses on the selection of source and destination node. In the second step it decides a unicast route from source to a particular destination. The results illustrates that energy consumption of this scheduling and routing algorithm is $50 \%$ less as compared to traditional scheduling and routing algorithm.

An-Ping Xiong [11] focused on three basic goals to reduce energy consumption in cloud environment. The main goals are 1) The quality of service can be minimized to a reasonable range to reduce the energy consumption. 2) Fix a maximum quantitative value for energy and then excerpt the maximum performance in that definite amount. 3) Negotiate the energy and performance parameters so that both are not compromised. The author follows the third criteria to perform VM consolidation based on virtualization technology using PSO algorithm. The results show that energy is reduced but the algorithm is limited to only two instances such as CPU and Disk. The additional resources such as network resources, memory etc is not taken into account.

Wanneng Shu et al. [12] proposed a new energy effective algorithm in which the resources are allocated based on immune clonal optimization method in a cloud computing environment. The cloudsim tool kit is used to analyze the performance of the algorithm. Time, cost and energy consumption are used in this algorithm. In experimental results it was found that in the proposed method, the response time and make span was significantly improved.

Yanwen Xiao et al [13] implemented a heuristic algorithm for data placement. In this heuristic method focuses on node scheduling which is used to reduce the energy consumption in a cloud environment. The main objective of this research is to accommodate maximum data on minimum number of nodes using Greedy approach. The algorithm is implemented using cloudsim.

Meenakshi Sharma and Pankaj Sharma [14] implemented a virtual machine load balancer algorithm. In this algorithm, Data Centre Controller uses a Virtual Machine Load Balancer to determine which VM should be allocated to the next Cloudlet for data processing. There are two Virtual Machine Load Balancers implementing policies which can be selected as required by the developer [14]. The Round-robin Load Balancer - uses a simple round-robin algorithm to allocate VMs. The Active Monitoring Load Balancer balances the load and assigns the tasks among available VM's in a way to even out the number of active tasks on each VM at any given time. The Round Robin Load Balancer selects the virtual machine randomly. The datacenter controller allocates the requests to a list of VMs on a circular order. The first request is allocated to a VM chosen arbitrarily from the group and then the Data Center controller allocates the requests in a circular order. Once the VM is assigned the request, the VM is moved to the end of the list.

Raj, G implemented the Equally Spread Current Execution Algorithm. This algorithm process handle with priorities and it distributes the load randomly by checking the size. It transfers the load to the lightly loaded virtual machine or handles that task easy and takes less time, and give maximize throughput. This algorithm uses the spread spectrum technique to balance the load of the job in hand into multiple virtual machines [15]

\section{PROPOSED RESPONSE TIME MINIMIZATION SCHEDULING ALGORITHM}

The proposed Response Time Minimization Approach is compared with Active VM load balancer algorithm. The main objective is to allocate the load to the available VM effectively. So this algorithm improves the request response time and minimizes delay time. Here, Virtual machine initiated at zero. If the $\mathrm{VM}$ already utilized then virtual machine will be added. In this method VM should have assigned at least one value on the load. If the selected VM is not in free mode then it will select other VM for execution from list. In this process, this approach have following three information namely request time, response time and processing time to evaluate request-response model between user base and datacenter. First, it evaluates request time since the last response time (datacenter) is determined and a COMPUTE (<seconds>) process is added to evaluate the approach. Second, the request-response operation and its arguments are added. Third, processing time information is added, as per user data request per/hr. When a request/response is being delayed, then approach delays issuing the request/response until all datacenter either not available or block.

A remote procedure request is sent from service provider to data center. If a user base is available in particular region, then it's not a cause of delayed request-response operation. Otherwise, data request user adds a (SIGNAL) to its evaluation of job waiting task. i.e how muck task is queue who are waiting for response from datacenter with details of user base and region. Once user base receive request then it verify the VM allocation, job scheduling and it process the request in datacenter to offer service to user. This approach response to user base with less processing time after getting acknowledgment form data center. 


\section{Pseudo code for Response Time Minimization scheduling}

Input: Users U, User's Region R, Data Size D, Datacenter DC, Virtual Machine VM, Request Grouping Factor RGF, User Grouping Factor UGF, and Execution Per Request EPF

\section{Procedure}

Enters $U$ and $\mathrm{D}$ details

Configure R, DC and VM with Memory $\mathrm{M}$ and Bandwidth BW

Select the Data Processing Allocation policy in DC

If $U$ enters the R, D, DC and VM Details then

Run the simulation

Else

Back to input parameters page

End if

Apply efficient scheduling

Configure the RGF, UGF, EPF and

Data Processing

Allocation policy then Proceed the simulations

If $\mathrm{VM}$ is equally distributed $\mathrm{D}$ then

Efficient scheduling reduce the RT and DCPT

Else

It display manually distributed $\mathrm{d}$ time

End if

Output: Response time RT, and Data center processing time DCPT.

Response Time Minimization Approach is the critical concept of the load balancing in a cloud computing environment. There is a greater need for load balancing in complex and large systems. For simplifying load balancing in a cloud, Round robin load balance random sampling method is used. The random sampling method selects the load randomly. Some server may be heavily loaded or some may be lightly loaded. To overcome the problem, the proposed method use ESCE load algorithm. ESCE distributes the current load to all servers.

\section{EXPERIMENTAL SETUP}

The Proposed Response time minimization task scheduling algorithm is implemented using Cloudanalyst tool [16]. Java language is used for implementing proposed scheduling algorithm. Assume that the application has been deployed in two data center. The data center is having 50 virtual machines (with $1024 \mathrm{Mb}$ of memory in each VM running on physical processors capable of speeds of 100 MIPS) where the parameter values are as under:

\subsection{Input Parameters}

The cloud provider can determine the best way to allocate resources by performing various simulations operation.

Table-01 Cloud Experimental Evaluation parameter Details

\begin{tabular}{|l|l|}
\hline \multicolumn{1}{|c|}{ Parameters } & Value \\
\hline User & 06 \\
\hline Region & 06 \\
\hline Datacenter & 2(DC1 and DC2) \\
\hline
\end{tabular}

\begin{tabular}{|l|l|}
\hline Virtual Machine & $50\left(\mathrm{DC}_{1}\right)$ and 50(DC 2$)$ \\
\hline Data Centre VM & Xen \\
\hline $\begin{array}{l}\text { Number of Process Machine } \\
\text { Wise }\end{array}$ & 16 \\
\hline Data Center Processing Speed & 100 MIPS \\
\hline Data Centre VM Policy & Time Shared \\
\hline Data Centre OS & Windows 7 \\
\hline VM Memory & 2048 \\
\hline Data Centre Architecture & X86 \\
\hline Bandwidth & 1000 Mbps \\
\hline User Grouping Factor & 1000 \\
\hline Request Grouping Factor & 100 \\
\hline Executable Length & 250 \\
\hline
\end{tabular}

\subsection{Cloudanalyst Tool}

The 'Response Time Minimization Algorithm' is executed using cloudanalyst tools, for the Datacenter to effectively load balance requests between the available virtual machines. The performance analysis indicates that the response time of the proposed task scheduling algorithm is much better than the Round Robin task scheduling algorithm and equally spread execution algorithm. The proposed Response Time Minimization Approach is compared with Round Robin Algorithm and equally spread execution algorithm. The cloudsim is a GUI based tool that allows doing modeling, simulation and other experimentation. The cloud analyst sets the location of users and data centers. In this configuration various parameters can be used. The parameters are number of users, number of request made per user per hour , number of virtual machines, number of processors, amount of storage, network bandwidth and other necessary parameters. Based on these parameters the tool calculates the simulation result and shows the results in graphical form [17]. The result includes response time and processing time.

\section{RESULTS AND DISCUSSIONS}

The proposed method is implemented using Cloudanalyst tool, and effectively balances the load in the available virtual machines. It uses the following Quality of service parameter namely response time, processing time, bandwidth utilization and delay time.

\subsection{Response Time}

In this section, proposed method represents mathematical model behalf of response time in equation (1). Here, Response time is calculated based every user base maximum and minimum response by proposed approach. Response time contains following factor $\mathrm{T}_{\text {Finish }}$, which time is taken data center to receive the request from user base. $\mathrm{T}_{\text {Arrival }}$, is recorded by datacenter, which request came. $\mathrm{T}_{\text {Delay }}$ is dealing out of the request to reach in datacenter. 


$$
T_{\text {Responsc }}=T_{\text {Finish }}-T_{\text {Arrival }}+T_{\text {Delay }}
$$

\subsection{Processing Time}

In this section, proposed method represents mathematical model with respect to Datacenter Processing Time is shown in equation (2). Here, datacenter processing take care the entire request which are given user in various regions. Hence, it processes for retrieve the requested query from database.

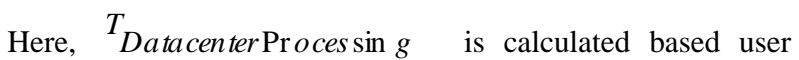
request per hour with respect of bandwidth allocation (1000 mbps).

$$
T_{\text {Datacenter } P r \text { oces } \sin g}=\frac{R}{B W_{\text {Userbase }}}
$$

\subsection{Bandwidth Utilization}

In this section, proposed method represents mathematical model respect of Bandwidth Utilization in equation (3). Here, bandwidth utilization depends on user request, data transfer size, and region. Proposed approach evaluates $\mathrm{BW}_{\text {Allocated, }}$ which done by cloud server before evaluation. $\mathrm{N}_{\mathrm{ur}}$ contains number of user request whose request is going to transmit the request in different-different regions. It also contains number user request between two regions.

$$
B W_{\text {utlization }}=\frac{B W_{\text {Allocation }}}{N_{u r}}
$$

\subsection{Delay Time}

In this section, proposed method represents mathematical model behalf of Delay Time in equation (4). In this subsection, delay time calculates the difference between request arrival time and response time i.e when user receive the response from datacenter. It also contains delay time between two regions when user is available in different location and datacenter in different locations. Here $\mathrm{T}_{\text {Finished }}$ represents total time taken to complete the task and $\mathrm{T}_{\text {Estimate }}$ represent assumption time which are assigned by analyzer before start the task. $\mathrm{T}_{\text {Arrived }}$ indicate the time when request reach to data center and $\mathrm{T}_{\text {Datacenter Processing }}$ indicate processing time.

$$
T_{\text {Delay }}=\left(T_{\text {Finished }}-T_{\text {Estimate }}\right)-\left(T_{\text {Arrived }}-T_{\text {Datacenter } \mathrm{Pr} \text { ocessing } g}\right)
$$

Table 2 shows the overall response time for Response time Minimization Task scheduling algorithm. Table 3 shows the Response Time by Region. Figure no. 1 to 6 represents User Base hourly Response time. Table 2 represents the overall response time summary and table 3 shows the response time by region. Table 4 comparative studies on proposed scheduling algorithm with Round robin and equally spread algorithm. Figure 7 shows the performance analysis of proposed algorithm with Round Robin and Equally spread execution. It shows that Response time is minimized compared to Round Robin and Equally Spread algorithm.

Table 2 Overall Response Time Summary:

\begin{tabular}{|l|c|c|c|}
\hline & Average (ms) & Min (ms) & Max (ms) \\
\hline $\begin{array}{l}\text { Overall } \\
\text { Response Time }\end{array}$ & 233.58 & 48.25 & 600.88 \\
\hline $\begin{array}{l}\text { Data Center } \\
\text { Processing Time }\end{array}$ & 34.60 & 2.20 & 133.31 \\
\hline
\end{tabular}

Table 3 Response Time by Region:

\begin{tabular}{|c|c|c|c|}
\hline User Base & Average (ms) & $\begin{array}{c}\text { Min } \\
(\mathbf{m s})\end{array}$ & Max (ms) \\
\hline UB1 & 57.02 & 48.25 & 66.49 \\
\hline UB2 & 98.02 & 73.83 & 145.55 \\
\hline UB3 & 329.92 & 248.93 & 411.08 \\
\hline UB4 & 118.75 & 66.73 & 187.19 \\
\hline UB5 & 520.87 & 398.19 & 600.88 \\
\hline UB6 & 206.95 & 174.96 & 239.82 \\
\hline
\end{tabular}

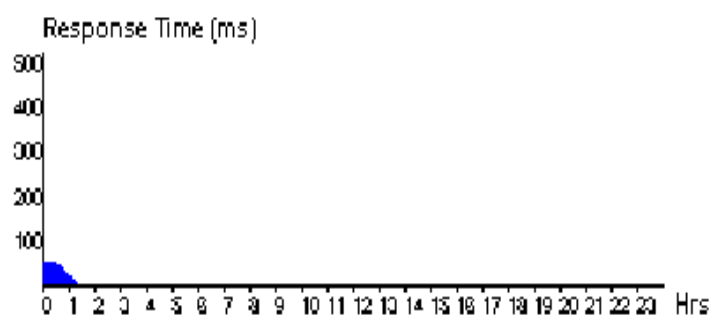

Figure 1 User Base Hourly Response Time (UB1)

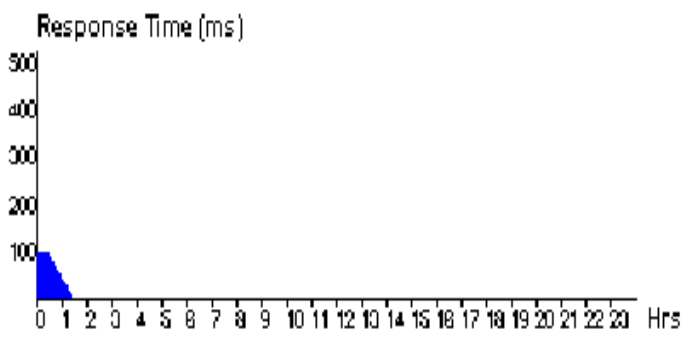

Figure 2 User Base Hourly Response Time (UB2)

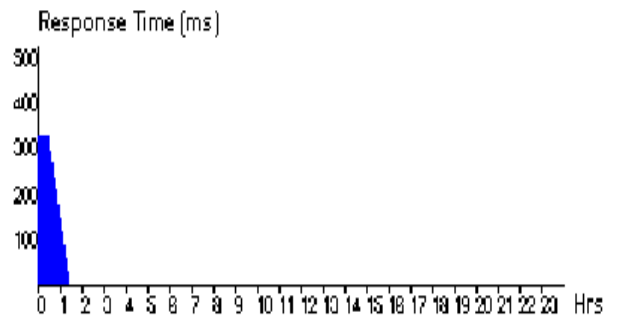

Figure 3 User Base Hourly Response Time (UB3) 


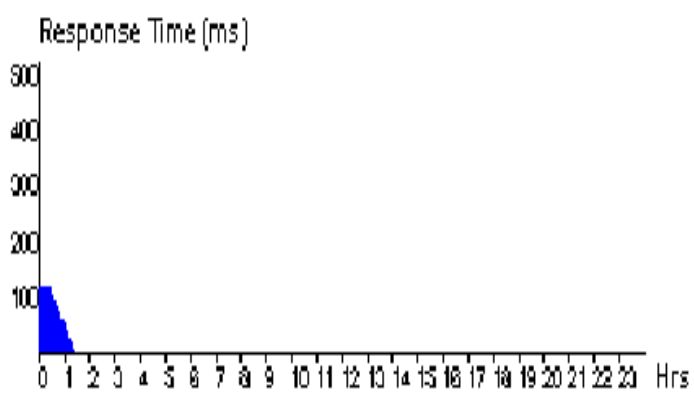

Figure 4 User Base Hourly Response Time (UB4) Responge Time (ms)

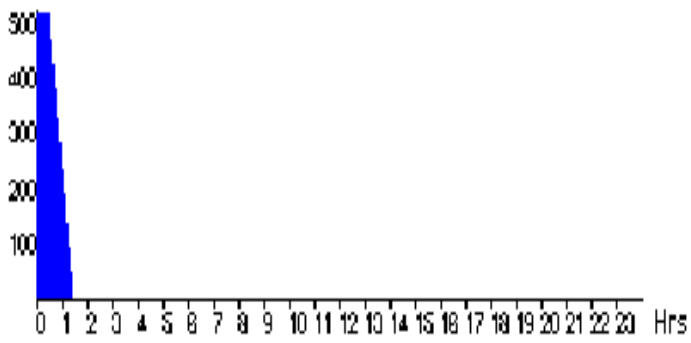

Figure 5 User Base Hourly Response Time (UB5 )

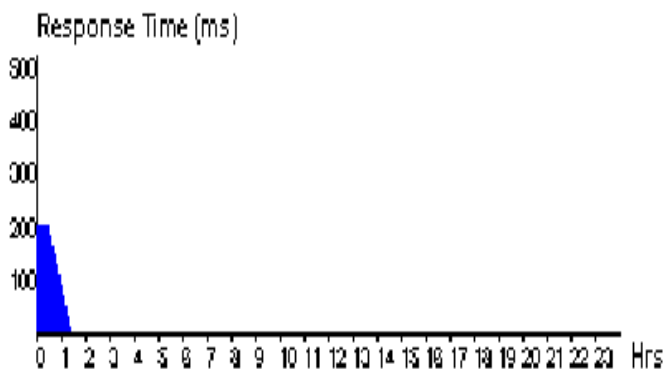

Figure 6 User Base Hourly Response Time (UB6)

Table 4 comparative studies on proposed scheduling algorithm with Round robin and equally spread algorithm

\begin{tabular}{|c|c|c|}
\hline Algorithm & $\begin{array}{c}\text { Response Time } \\
(\mathbf{m s})\end{array}$ & $\begin{array}{c}\text { Data Center } \\
\text { Processing Time } \\
(\mathbf{m s})\end{array}$ \\
\hline $\begin{array}{c}\text { Response Time } \\
\text { Minimization }\end{array}$ & 233.58 & 34.6 \\
\hline $\begin{array}{c}\text { Equally spread } \\
\text { Execution }\end{array}$ & 248.13 & 49.27 \\
\hline Round Robin & 244.51 & 45.57 \\
\hline
\end{tabular}

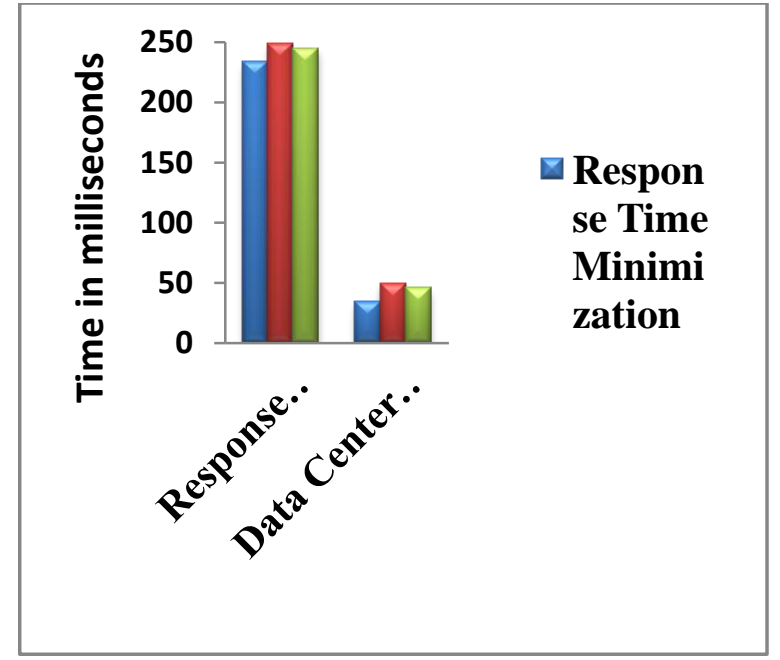

Figure 7 performance analysis of proposed algorithmwith Round Robin and Equally spread execution.

\section{CONCLUSION}

The Response time minimization algorithm is used to share the load to the available VM efficiently. It improves the response time and minimizes the delay time. Hence the load balancing is achieved. Several performance metrics such as availability, utilization, and responsiveness used to investigate the impact of different strategies on both provider and user point of views. In a market-oriented area, such as the cloud computing, a precise evaluation of these parameters is required to quantify the offered QoS and opportunely manage SLAs. This paper identifies that Response Time Minimization grid task scheduling algorithm outperforms the Round Robin and Equally spread algorithm in a heterogeneous distributed environment. The experimental results shows that Response time is minimized compared to Round Robin and Equally Spread algorithm.This study can be further extended by proposing a new hybrid algorithm to represent PaaS and SaaS cloud systems and to integrate the mechanisms to capture VM migration and data center consolidation aspects for energy saving policies.

\section{REFERENCES}

[1] B.Santhosh Kumar, Latha Parthiban (2015). An Energy Efficient Data Center Selection Framework for virtualized Cloud Computing Environment. Indian Journal of Science and Technology, vol 8(35). ISSN (Print) : 0974-6846

[2] Pushtikant Malviya, Swapnamukta Agrawal, Shailendra Singh, "An Effective Approach for Allocating VMs to Reduce the Power Consumption of Virtualized Cloud Environment," Fourth International Conference on Communication Systems and Network Technologies, IEEE, 2014

[3] Akhil Goyal, Bharti, "A Study of Load Balancing in Cloud Computing using soft Computing Techniques," International Journal of Computer Applications (c) 2014 by IJCA Journal, Vol. 92, No. 9, 2014.

[4] Hemamalini M. (2012). Review on grid task scheduling algorithm in a distributed heterogeneous environment. International Journal of Computer Applications. 40(2):24-30.

[5] M.Hemamalini, Dr.M.V.Srinath "State of the Art: Task Scheduling Algorithms in Heterogeneous Grid 
Computing Environment", Elysium Journal of Engineering Research and Management, Volume-1, Issue-1, August 2014.

[6] Kokilavani T, Amalarethinam GDI. Load balanced minmin static meta-task scheduling algorithm in a grid computing environment. International Journal of Computer Applications. 2011; 20(2):43-9.

[7] Hemamalini M. Dr.M.V.Srinath. (2015).Memory Constrained Load Shared Minimum Execution Time Grid Task Scheduling Algorithm in a Heterogeneous Environment. Indian Journal of Science and Technology, Vol 8(15), ISSN (Print) : 0974-6846, ISSN (Online) : 0974-5645.

[8] Anton Beloglozov, Jemal Abawajy, Rajkumar Buyya,"Energy-aware Resource Allocation Heuristics for Efficient management of Data centers for Cloud Computing," Future Generation Computer Systems, vol.28, pp. 755-768, ELSEVIER 2012.

[9] Bharti Wadhwa, Amandeep Verma, "Energy Saving approaches for Green Cloud Computing: A Review," Proceedings of RAECS UIET Panjab University Chandigarh, IEEE, 06 - 08 March, 2014.

[10] Jens Buysse, Konstantinos Georgakilas, Anna Tzanakaki,Marc De Leenheer, Bart Dhoedt, Chris Develder, "Energy- Efficient Resource-Provisioning Algorithm for Optical Clouds," Journal of Optical Communication Network, Vol. 5, No. 3, pp. 226-239, March 2013

[11] An-ping Xiong, Chun-xiang Xu, "Energy Efficient Multiresource Allocation of Virtual Machine Based on PSO in Cloud Data Center," Hindawi Publishing Corporation, Mathematical Problems in Engineering,Vol. 2014, Article ID 816518, June, 2014.

[12] Wanneng Shu, Wei Wang, Yunji Wang, "A Novel Energy-Efficient Resource Allocation Algorithm based on Immune Clonal Optimization for Green Cloud Computing," EURASIP Journal on Wireless Communications and Networking, Springer, 2014.

[13] Yanwen Xiao, Jinbao Wang, Yaping li, Hong Gao, "An Energy-Efficient Data Placement Algorithm and Node Scheduling Strategies in Cloud Computing Systems," 2nd International Conference on Advances in Computer Science and Engineering, Atlantis Press (CSE 2013).

[14] Meenakshi Sharma , Pankaj Sharma ,"Performance Evaluation of Adaptive Virtual Machine Load Balancing Algorithm “, (IJACSA) International Journal of Advanced Computer Science and Applications, Vol. 3, No.2, 2012.

[15] Raj, G. (2012). Comparative Analysis of Load Balancing Algorithms in Cloud Computing, 1(3), 120-124.

[16] Bhathiya Wickremasinghe, Rodrigo N. Calheiros, and Rajkumar Buyya. "Cloud Analyst: A Cloud Sim-based Visual Modeller for Analysing Cloud Computing Environments and Applications", 20-23 April 2010 Page No 446-452.

[17] Tanveer Ahmed, Yogendra Singh (2012), Analytic Study of Load Balancing Technique Using Tool Cloud Analyst. International Journal of Engineering Research and Applications (IJERA). Vol. 2, Issue 2,Mar-Apr 2012, pp.1027-1030. ISSN: 2248-9622 .

\section{AUTHOR PROFILE}

Mrs.M.Hemamalini working as Assistant Professor in Department of computer science, A.V.C College, Mannampandal, Mayiladuthurai. She has obtained Masters Degree from Bharathidasan University, Tiruchirapalli. Currently doing research in the field of Grid Computing. She has presented papers in National conferences and International conferences. She has also published papers in International journals. Her research interests include Parallel Computing, Grid Computing, Mobile Computing ,Cloud Computing and Data Mining.

Dr.M.V.Srinath,Currently Research Supervisor Bharathidasan University Trichy, India. He has published more than 100 Journals in National and International level and he also presented more than 60 papers in the National and International conferences. He has delivered more than 100 key note addresses and invited talks to different Universities and Colleges. He is a member of different Professional bodies like ISTE, CSI, ISTD, Member -World Council for Curriculum and Instruction. 